\title{
O papel do agente comunitário de saúde na disseminação de informações acerca dos principais cânceres de interesse na atenção básica
}

\author{
The role of community health worker in disseminating information about the main cancers of
} interest in basic care

El papel del agente comunitario de salud en la difusión de información sobre los principales cánceres de interés en atención básica

Recebido: 28/12/2020 | Revisado: 29/12/2020 |Aceito: 08/01/2021 | Publicado: 11/01/2021

\author{
Alex Luiz Menezes da Silva \\ ORCID: https://orcid.org/0000-0002-7878-3450 \\ Universidade Federal do Pará, Brasil \\ E-mail: aalexmenezess@gmail.com \\ Weydder Tavares da Silva \\ ORCID: https://orcid.org/0000-0002-8683-2371 \\ Universidade Federal do Pará, Brasil \\ E-mail: weyddert@gmail.com \\ Eliel Barbosa Teixeira \\ ORCID: https://orcid.org/0000-0001-5102-1811 \\ Universidade Federal do Pará, Brasil \\ E-mail: elielcn2015@gmail.com \\ André Salim Khayat \\ ORCID: https://orcid.org/0000-0002-3451-6369 \\ Universidade Federal do Pará, Brasil \\ Email: andreskh@gmail.com \\ Thaíssa Vitória Portal Rodrigues \\ ORCID: https://orcid.org/0000-0001-8912-9369 \\ Universidade Federal do Pará, Brasil \\ E-mail: thaissa.rodrigues@icb.ufpa.br \\ Bruna Cláudia Meireles Khayat \\ ORCID: https://orcid.org/0000-0002-3962-1483 \\ Universidade Federal do Pará, Brasil \\ E-mail: brunamk@gmail.com
}

\begin{abstract}
Resumo
Este trabalho tem por objetivo avaliar os conhecimentos prévios dos agentes comunitários de saúde (ACS) atuantes em Belém do Pará sobre os principais tipos de câncer na atenção básica (AB). Para isso, desenvolveu-se uma pesquisa transversal, descritiva, em que participaram 66 ACS, de seis unidades de saúde da família do distrito administrativo do Guamá. A fim de avaliar os conhecimentos, aplicou-se um questionário com questões objetivas abordando os eixos: socioeconômicos e demográficos dos participantes; conhecimentos prévios sobre a fisiopatologia do câncer; prevenção e fatores de riscos dos principais cânceres na $\mathrm{AB}$ e níveis de organização de Atenção em Oncologia. Entre os resultados, constatou-se que a maioria dos pesquisados conheciam a fisiopatologia, assim como a definição correta de metástase $(87,88 \%)$. Sobre o aspecto de prevenção do câncer, $95,45 \%$ apontaram medidas ou fatores de riscos que estão relacionados ao desenvolvimento da doença. Contudo, poucos distinguiam os níveis da atenção especializada em oncologia e suas atribuições; assim como a periodicidade e faixa etária envolvida nos rastreios do câncer de mama $(27,27 \%)$ e colo de útero $(30,30 \%)$, ou seja, apenas uma minoria afirmou conhecer a conduta adequada para rastreio do câncer de mama e colo do útero de acordo com o Ministério da Saúde. Além disso, ter familiar com câncer apresentou relevância estatística quanto ao número de assertivas corretas $(p<0,05)$. Conclui-se que, possivelmente, existam deficiências no conhecimento dos ACS sobre tal temática, com percentual de acerto maior nos que tiveram parentes acometidos com algum tipo de câncer.
\end{abstract}

Palavras-chave: Oncologia; Agentes comunitários de saúde; Atenção primária à saúde.

\begin{abstract}
This work aims to evaluate the previous knowledge of community health workers (CHW) working in Belém-Pará on the main types of cancer in primary care (PC). For this, a cross-sectional, descriptive study was carried out, in which 66 CHW participated, from six family health units in the administrative district of Guamá. In order to assess knowledge, a questionnaire was applied with objective questions addressing the axes: socioeconomic and demographic of the participants; previous knowledge about the pathophysiology of cancer; prevention and risk factors of major cancers in PC and levels of organization of Oncology Care. Among the results, it was found that most
\end{abstract}


respondents knew the pathophysiology, as well as the correct definition of metastasis (87.88\%). Regarding the cancer prevention aspect, $95.45 \%$ indicated measures or risk factors that are related to the development of the disease. However, few distinguished the levels of specialized care in oncology and their duties; as well as the periodicity and age group involved in screening for breast cancer $(27.27 \%)$ and cervix $(30.30 \%)$, that is, only a minority claimed to know the proper conduct for screening breast and cervix cancer according to the Ministry of Health. In addition, having a family member with cancer was statistically relevant as to the number of correct statements $(p<0.05)$. It is concluded that, possibly, there are deficiencies in the knowledge of CHW on this theme, with a higher percentage of correct answers in those who had relatives affected with some type of cancer.

Keywords: Medical oncology; Community health workers; Primary health care.

\section{Resumen}

Este trabajo tiene como objetivo evaluar el conocimiento previo de los agentes comunitarios de salud (ACS) que trabajan en Belém-Pará sobre los principales tipos de cáncer en atención primaria (AP). Luego, se realizó una investigación descriptiva transversal con $66 \mathrm{ACS}$, de seis unidades de salud familiar en el distrito administrativo de Guamá. Para evaluar el conocimiento se aplicó un cuestionario con preguntas objetivas que abordan los ejes: socioeconómico y demográfico del participante; conocimientos previos sobre la físiopatología del cáncer; prevención y factores de riesgo de cánceres mayores en AP y niveles de organización de la Atención Oncológica. Como resultado, se encontró que la mayoría de los encuestados conocían la fisiopatología, así como la correcta definición de metástasis $(87,88 \%)$. Sobre la prevención del cáncer, 95,45\% indicó medidas o factores de riesgo que se relacionan con el desarrollo de la enfermedad. Pero, pocos distinguieron los niveles de atención especializada en oncología y sus funciones; así como la periodicidad y el grupo de edad involucrados en el cribado de cáncer de mama $(27,27 \%)$ y de cérvix $(30,30 \%)$, es decir, solo una minoría afirmó conocer la conducta adecuada para el cribado de cáncer de mama y cérvix según el Ministerio de Salud. Además, tener un familiar con cáncer fue estadísticamente relevante en cuanto al número de afirmaciones correctas $(\mathrm{p}<0,05)$. Se concluye que, posiblemente, existen deficiencias en el conocimiento de los ACS sobre este tema, con un mayor porcentaje de aciertos en quienes tenían familiares afectados por algún tipo de cáncer.

Palabras clave: Oncología médica; Agentes comunitarios de salud; Atención primaria de salud.

\section{Introdução}

A palavra câncer designa um grupo de doenças heterogêneas, que compartilham o crescimento desordenado de células (Instituto Nacional do Câncer -INCA, 2020. p.13). Segundo a Organização Mundial da Saúde (OMS, 2019) é a segunda causa de mortes ao redor do mundo, responsável por cerca de 9,6 milhões de mortes em 2018. Atualmente, essa doença é responsável por 1 em cada 7 mortes em todo o mundo (American Cancer Society, 2019).

No Brasil, o INCA (2019, p.120) estima para o triênio 2020-2022 a incidência de 625 mil casos de câncer para cada ano, com exceção do câncer de pele não melanoma, mais comum em ambos os sexos. Os tipos mais frequentes em homens são próstata $(29,2 \%)$, cólon e reto $(9,1 \%)$ e pulmão $(7,9 \%)$; enquanto nas mulheres, mama $(29,7 \%)$, cólon e reto $(9,2 \%)$ e colo do útero $(7,4 \%)$. Tal fenômeno corresponde ao aumento da morbidade por doenças crônicas não transmissíveis, acometendo principalmente a população idosa. Esse cenário se justifica pela maior exposição a agentes cancerígenos e aumento da expectativa de vida, INCA (2020, p.13).

Diante deste problema, a Atenção Básica $(\mathrm{AB})$ se configura como importante estratégia para o diagnóstico precoce e promoção da saúde, principalmente no desenvolvimento de ações de educação em saúde. Segundo a Política Nacional da Atenção Básica (PNAB), a $\mathrm{AB}$ é o conjunto de ações de saúde que visam a promoção, prevenção, proteção, diagnóstico, tratamento, reabilitação, redução de danos, cuidados paliativos e vigilância em saúde, por meio de atividades desenvolvidas por equipes multiprofissionais, incluindo os Agentes Comunitários de Saúde (ACS). Além disso, caracteriza-se por ser a principal porta de entrada e comunicação da Rede de Atenção à Saúde (Brasil, 2017). Com papel especial aos ACS, principalmente no desenvolvimento de ações de educação em saúde, visto que convivem rotineiramente com as famílias (Gomes, 2011; Almeida, 2013).

Alguns estudos avaliaram o conhecimento dos ACS sobre determinadas doenças, como o HPV (Papiloma Vírus Humano), vírus relacionado ao desenvolvimento do câncer do colo uterino, em que se evidenciou um escasso conhecimento sobre esta patologia, podendo repercutir negativamente nas atuações como agentes disseminadores de prevenção e promoção 
em saúde (Manoel, Rodrigues, Piva, Warpechowski \& Schuelter-Trevisol, 2017). Resultado semelhante foi encontrado ao avaliar conhecimentos e práticas relacionadas ao câncer do colo do útero por profissionais de saúde, incluindo médicos, enfermeiros e ACS no Brasil, destaca-se que o grupo de ACS foram os menos propensos a afirmar possuir conhecimento adequado das diretrizes relacionadas ao rastreamento deste câncer (Stormo, Moura \& Saraiya, 2014).

Geralmente, esses profissionais são admitidos por meio de processos seletivos. Na Prefeitura de Belém do Pará, apresentam vínculos celetistas, conforme a lei 9.218/2016, que regulamenta e cria os empregos públicos de ACS e Agente de Combate às Endemias. Outrossim, o exercício da atividade se dá mediante o cumprimento de alguns requisitos, entre eles, a conclusão de um curso de formação. Ou seja, o cargo exige uma formação prévia dos candidatos sobre assuntos relacionados à saúde da comunidade, no entanto esse curso ofertado acaba sendo pouco abrangente e curto, por isso, uma formação continuada é importante para esses agentes.

Por conseguinte, este trabalho tem por objetivo avaliar os conhecimentos prévios dos Agentes Comunitários de Saúde (ACS) atuantes em Belém do Pará sobre os principais tipos de câncer na atenção básica.

\section{Metodologia}

Este trabalho se caracteriza como um estudo descritivo, de natureza qualitativa, visto que o foco principal desse tipo de estudo é a importância que os entrevistados dão ao assunto em questão, e os dados obtidos não podem ser expressos em grandezas, embora possam ser tratados como categóricos, no momento de uma futura análise estatística, conforme Pereira, Shitsuka, Parreira e Shitsuka (2018). Observou-se a resolução nº 466/2012 do Conselho Nacional de Saúde, sendo submetido e aprovado pelo Comitê de Ética em Pesquisa do Núcleo de Pesquisa em Oncologia, da Universidade Federal do Pará, sob o número do parecer 3.290.217, de abril de 2019.

A amostragem foi não probabilística por conveniência, sendo incluídos apenas os ACS, de qualquer grau de escolaridade e idade, que aceitaram os termos do estudo. Atuantes nas seguintes Unidades de Saúde da Família (USF): Condor, Parque Amazônia I e II, Radional, Riacho Doce e Terra Firme. Estas pertencentes ao Distrito Administrativo do Guamá (DAGUA) em Belém do Pará, totalizaram 102 agentes comunitários contratados em abril de 2019, destes 66 foram voluntários da pesquisa. O município contava com total de 645 servidores remunerados pela Secretaria Municipal de Saúde atuantes como ACS, sendo 102 pertencentes ao DAGUA. Pesquisa executada no período de março a julho de 2019.

A coleta de dados foi realizada por meio de questionário avaliativo de própria autoria, objetivo, não validado previamente, com questões de múltipla escolha, contendo itens para julgar como verdadeiro ou falso ou correlacionar, e perguntas com única alternativa correta ou de caixas de seleção, em que poderiam marcar mais de uma alternativa. As aplicações dos questionários ocorreram após a autorização dos gestores das USF. Estes reservavam uma sala dentro das unidades, onde os pesquisadores entregavam o questionário para cada ACS responder de forma individual. A confecção e aplicação do questionário foi realizada pelos pesquisadores, sob a supervisão dos docentes autores deste trabalho, utilizando como referência o livro “ABC do Câncer - Abordagens Básicas para o Controle do Câncer”, 4 edição (2018), do Instituto Nacional do Câncer José Alencar Gomes da Silva.

As questões apresentavam os seguintes eixos: perfil socioeconômico e demográfico; conhecimentos prévios sobre a fisiopatologia, prevenção e fatores de riscos do câncer; níveis de organização da Atenção em Oncologia; rastreio para câncer de mama e colo do útero; e sintomas do câncer de próstata. Este questionário se encontra no repositório de dados Zenodo, DOI 10.5281/zenodo.3763764; não sendo compartilhado os dados anonimizados dos participantes.

As variáveis analisadas foram gênero, idade, etnia, estado civil, renda familiar, escolaridade, histórico de câncer familiar; conhecimentos sobre câncer de mama, próstata e útero, além de aspectos da fisiopatologia, prevenção e atenção especializada em oncologia. Os dados extraídos dos questionários foram organizados no programa Excel 2013, e para fins 
estatísticos como variáveis categóricas, atribuindo para os resultados dicotômicos os números: 0 e 1, quando possível. O teste estatístico utilizado foi qui-quadrado $\left(\mathrm{X}^{2}\right)$ que é o mais indicado nesses casos, no programa Bioestat, versão 5.3 (AYRES et al. 2007), considerando significância de p<0,05, IC:95\%.

\section{Resultados}

Participaram da pesquisa 66 ACS, representando 10,23\% do total de ACS do município e 64,7\% do distrito. A distribuição dos participantes foi de 25,76\% $(n=17)$ pertencentes a USF- Terra Firme, 24,24\% ( $n=16)$ USF- Radional, 16,67\% ( $n=11)$ USF- Condor, 12,12\% (n=8) USF- Parque Amazônia II e Riacho Doce, e 9,09\% (n=6) USF- Parque Amazônia I.

Os voluntários tinham média de 39,32 anos de idade (DP \pm 7.09 ), sendo majoritariamente do sexo feminino, pardos, solteiros e naturais de Belém. Quanto ao aspecto socioeconômico, a maioria eram de moradores da área urbana, com renda familiar entre 1 e 2 salários mínimos, e grau de escolaridade até o ensino médio completo (Tabela 1). Ao serem indagados sobre histórico de câncer familiar, a maioria apresentou algum parente acometido com tal enfermidade (Tabela 1), sendo o local mais frequente o útero $(33,33 \% / n=12)$, seguido por estômago $(25 \%$ / n=9) e próstata $(11,11 \%$ / n=4). Os avós foram os familiares mais atingidos $(30,56 \% / \mathrm{n}=11)$.

Tabela 1 - Caracterização demográfica, socioeconômica e histórico de câncer familiar dos voluntários.

\begin{tabular}{|c|c|c|}
\hline \multirow{2}{*}{ Variáveis } & \multicolumn{2}{|c|}{ Total } \\
\hline & $\mathbf{n}$ & $\%$ \\
\hline \multicolumn{3}{|l|}{ Demográficas } \\
\hline \multicolumn{3}{|l|}{ Sexo } \\
\hline Feminino & 52 & 79 \\
\hline Masculino & 14 & 21 \\
\hline \multicolumn{3}{|l|}{ Faixa Etária } \\
\hline $20-29$ & 2 & 3 \\
\hline $30-39$ & 36 & 55 \\
\hline $40-49$ & 20 & 30 \\
\hline $50-59$ & 8 & 12 \\
\hline \multicolumn{3}{|l|}{ Raça/Cor } \\
\hline Amarela & 1 & 2 \\
\hline Branca & 4 & 6 \\
\hline Indígena & 1 & 2 \\
\hline Negra & 10 & 15 \\
\hline Parda & 48 & 73 \\
\hline Não informado & 2 & 3 \\
\hline \multicolumn{3}{|l|}{ Estado Civil } \\
\hline Casado (a) & 25 & 38 \\
\hline Divorciado (a) & 1 & 2 \\
\hline Solteiro (a) & 37 & 56 \\
\hline União Estável & 3 & 5 \\
\hline Naturalidade & & \\
\hline
\end{tabular}




$\begin{array}{lcc}\text { Pará } & & \\ \quad \text { Belém } & 46 & 70 \\ \quad \text { Interior } & 17 & 26 \\ \text { Outro Estado } & 3 & 5 \\ \text { Residência } & & \\ \quad \text { Rural } & 1 & 2 \\ \text { Urbana } & 57 & 86 \\ \text { Não informado } & 8 & 12\end{array}$

\section{Socioeconômicas}

Escolaridade

$\begin{array}{lcc}\text { E. médio completo } & 41 & 62 \\ \text { E. médio incompleto } & 3 & 5 \\ \text { E. Superior completo } & 12 & 18 \\ \text { E. Superior incompleto } & 8 & 12 \\ \text { Não informado } & 2 & 3\end{array}$

Renda Familiar

Entre 1 e 2 salários mínimos $\quad 53 \quad 80$

Entre 3 e 5 salários mínimos $\quad 11 \quad 17$

Menor que 1 salário mínimo $1 \quad 2$

Não informado $\quad 1 \quad 2$

\section{Câncer Familiar}

\begin{tabular}{lcc} 
Não & 29 & 44 \\
Sim & 36 & 55 \\
Não informado & 1 & 2 \\
\hline
\end{tabular}

Fonte: Protocolo de Pesquisa (2019).

Em relação aos conhecimentos sobre a fisiopatologia do câncer, com perguntas de verdadeiro ou falso, observou-se que $92,42 \%$ (n=61) entendiam que as células cancerígenas podem invadir outros órgãos e/ou tecidos vizinhos, sendo fruto de alterações cumulativas no DNA $(74,24 \%$ / n=49); e com o crescimento diferente das células normais $(81,82 \%$ / $n=54)$, pois ao invés de morrerem, continuam crescendo incontrolavelmente formando novas células anormais $(90,91 \% / \mathrm{n}=60)$. A maioria $(75,76 \%$ / n $=50)$ relacionou os tumores benignos com células que crescem lentamente, sendo semelhantes as suas células vizinhas; e tendem a permanecer com as mesmas funções da célula original $(50 \%$ / $n=33)$. Para 87,88\% ( $\mathrm{n}=58$ ) o termo metástase se refere à disseminação do câncer para outras áreas do corpo, sendo que esta disseminação não é exclusivamente feita pela via sanguínea $(81,82 \%$ / n=54). Por fim, 75,78\% (n=50) associaram o surgimento do câncer a falha nos mecanismos do sistema de defesa, reparação ou destruição celular; e 74,24\% (n=49) não o consideraram uma enfermidade contagiosa.

Sobre a prevenção do câncer, 95,45\% (n=63) relacionaram-no com um conjunto de medidas que reduzem ou evitam a exposição a fatores que aumentem a possibilidade de um indivíduo desenvolver tal agravo. Para 92,42\% (n=61) as causas para o desenvolvimento dessa doença são variadas, podendo ser internas ou externas ao organismo, estando ambas interrelacionadas. 
Na Tabela 2 são descritos os fatores de risco elencados pelos ACS como possíveis causadores de neoplasias. No questionário, esta era uma pergunta de múltipla escolha, devendo-se assinalar uma ou mais alternativas que apresentassem carcinógenos.

Tabela 2 - Fatores de risco apontados pelos agentes comunitários de saúde para desenvolvimento de neoplasias.

\begin{tabular}{lcc}
\hline \multirow{2}{*}{\multicolumn{1}{c}{ Fator de Risco }} & \multicolumn{2}{c}{ TOTAL } \\
& n & \% \\
\hline Tabaco & 64 & 97,0 \\
Agrotóxico & 61 & 92,4 \\
Bebidas Alcoólicas & 59 & 89,4 \\
Radiação Ultravioleta & 59 & 89,4 \\
Radiação Ionizante & 58 & 87,9 \\
Alimentação rica em gordura Saturada & 57 & 86,4 \\
Ter parceiro sexual com múltiplas parceiras & 52 & 78,8 \\
Obesidade & 52 & 78,8 \\
Sedentarismo & 50 & 75,8 \\
Possuir múltiplos parceiros sexuais & 49 & 74,2 \\
Agentes Infecciosos & 41 & 62,1 \\
Alimentação pobre em frutas & 40 & 60,6 \\
Iniciar precocemente as atividades sexuais & 37 & 56,1 \\
Exposições Ocupacionais & 33 & 50,0 \\
Poeira de Madeira & 33 & 50,0 \\
Poeira de Couro & 28 & 42,4 \\
Comportamento Sexual & 25 & 37,9 \\
Sílica & 22 & 33,3 \\
Asbesto & 21 & 31,8 \\
Não Soube Informar & 2 & 3,0 \\
\hline
\end{tabular}

Fonte: Protocolo de Pesquisa (2019).

Além disso, perguntou-se também sobre os níveis de organização da atenção oncológica, em que $65,15 \%$ (n=43) afirmaram desconhecer o significado de Unidade de Assistência de Alta Complexidade em Oncologia (UNACON), 72,72\% $(n=48)$ de Centro de Assistência em Alta Complexidade em Oncologia (CACON) e 69,70\% (n=46) Centro de Referência de Alta Complexidade em Oncologia (CRACON). Contudo, ao se pedir para correlacionar o significado de cada organização com sua respectiva função, apenas 4,55\% $(n=3)$ souberam o significado de UNACON, 13,66\% (n=9) o de CACON e 6,06\% $(n=4)$ de CRACON.

Em relação ao câncer de mama, 83,33\% (n=55) relacionaram a neoplasia a ambos os sexos; e 27,27\% (n=18) souberam rastrear de acordo com as diretrizes do Ministério da Saúde e ou INCA, avaliando-se apenas a faixa etária e periodicidade do rastreio. Em relação ao câncer de colo de útero, apenas $30.30 \%(n=20)$ souberam rastrear de forma adequada, e 83.33\% (n=55) correlacionaram o PCCU (Preventivo de Câncer do Colo do Útero) ao exame de investigação.

Quanto ao câncer de próstata, foram descritos os sinais e sintomas mais frequentemente associados: presença de sangue na urina $(84,85 \% / \mathrm{n}=56)$; dor ou queimação ao urinar $(60,61 \% / \mathrm{n}=40)$, aumento de um testículo $(57,58 \%$ / $\mathrm{n}=38)$, jato 
urinário fraco $(56,06 \% / \mathrm{n}=37)$, necessidade frequente de urinar à noite $(48.48 \%$ / n=32), e mudança nos hábitos intestinais $(18.18 \% / \mathrm{n}=12)$.

Finalmente, foram realizados testes de qui-quadrado em tabulações cruzadas $2 \times 2$ com intuito de verificar a associação das variáveis gênero, estado civil, renda familiar, grau de escolaridade, idade e câncer familiar quanto ao nível de conhecimento dos entrevistados sobre a temática câncer. Observou-se que apenas a variável câncer familiar apresentou influência significativa na quantidade de assertivas corretas no questionário.

Com um $\mathrm{p}<0,05$, neste estudo é possível concluir que existe uma associação significativa entre ter um parente com câncer familiar e o nível de informação do grupo amostral, visto que é possível notar um número maior de acertos por pessoas em tal situação. Ademais, a fim de melhor caracterizar essa associação, foi utilizado o OR (Odds Ratio), que é chance de um evento ocorrer em determinado grupo estudado. Ao analisar esses dados, observamos que pessoas com histórico de câncer familiar apresentaram 6,067\% de chances de acertarem as alternativas pré-definidas pelo questionário aplicado, IC 95\% $=$ $(2,084: 17,660) ; \mathrm{p}<0,05$.

\section{Discussão}

No cenário nacional o câncer ganha destaque como um problema de saúde pública (INCA, 2019, p.120). Para tanto, necessita-se disseminar conhecimentos sobre tal doença. Sabe-se que o câncer envolve a interação de fatores genéticos e ambientais (Hanahan \& Weinberg, 2011), relacionados ao estilo de vida, como a alimentação e exposição a agentes carcinógenos causadores de alterações genéticas, que propiciam o câncer (Vogelstein et al., 2013).

A educação em saúde na $\mathrm{AB}$ proporciona uma oportunidade para difundir conhecimentos em conjunto com o sistema de saúde. Fato confirmado no trabalho de Rodrigues et al. (2012) de prevenção do câncer do colo-uterino, em que foram esclarecidos os questionamentos sobre a temática, com importância na formulação de um novo conhecimento de promoção da saúde, utilizado na AB. Em outro estudo de Silva et al. (2011) envolvendo diagnóstico de câncer de mama, ações de intervenção educacional proporcionaram um aumento significativo na identificação precoce de mulheres em risco, contribuindo para o combate em estágio inicial da doença. Igualmente, observa-se em capacitações para rastreamento de câncer de colo de útero, havendo melhoras no preenchimento de formulários de requisição de exames citopatológicos, anamneses, exame clínico e da solicitação de exames para cada faixa etária (Amaral et al., 2014).

No presente estudo, os voluntários apresentaram conhecimento sobre a maioria dos fatores de riscos e fisiopatologia do câncer, sendo importantes para prática profissional. Dantas et al. (2018) avaliou o conhecimento dos ACS sobre o câncer de próstata, ressaltando a importância de ter agentes ativos na promoção da saúde integral e no compartilhamento de informações sobre o tema com a comunidade assistida. E advertiu para a necessidade de capacitação dos ACS na promoção da saúde, sobretudo em temáticas como a abordada nesta pesquisa.

Entretanto, observou-se deficiências no conhecimento sobre níveis de organização da atenção oncológica, periodicidade de rastreio dos principais cânceres femininos, sinais e sintomas do câncer de próstata e certos fatores de risco, como poeira de couro entre outros, observados na Tabela 2. Tais deficiências prejudicam a atuação do profissional na prevenção e detecção precoce do câncer na $\mathrm{AB}$ de Saúde.

Este ponto de vista é corroborado pelas atribuições específicas do ACS mencionadas na PNAB (Brasil, Ministério da Saúde, Secretaria de Atenção à Saúde e Departamento de Atenção Básica, 2012), "Orientar as famílias quanto à utilização dos serviços de saúde disponíveis”, e "desenvolver atividades de promoção da saúde, prevenção de doenças e agravos e de vigilância à saúde”. Dessa forma, notou-se prejuízos na identificação de comportamentos de risco, esperando-se que o ACS tenha dificuldades em levar informações à equipe de saúde e em conjunto atuarem no problema (Brito, 2016).

Somando-se a isso, dificuldades na orientação quanto aos serviços de saúde, pois poucos sabiam o significado de 
UNACON (4,55\%) ou CACON (13,66\%). De forma geral, a UNACON é o estabelecimento de saúde capaz de prestar serviços especializados em oncologia relativa aos cânceres mais prevalentes no Brasil, enquanto o CACON seria de todos os tipos de câncer, incluindo hematológicos (Ministério Da Saúde, 2014). De acordo com o site do INCA (2019) ambos presentes no município, sendo o Hospital Ofir Loyola um CACON, e os Hospitais Oncológico Infantil Octávio Lobo e Universitário João de Barros Barreto exemplos de UNACON. Quanto ao CRACON não existe tal serviço no estado.

Sobre o câncer de colo de útero, Santos e Varela (2015) assinalaram como principais entraves para não realização do exame de rastreio o desconhecimento sobre a importância da prevenção, medo do exame e resultado, dificuldades de acesso a unidade de saúde e negligência dos profissionais quanto orientação/informação sobre o câncer, sua prevenção e a importância do exame. Tais pontos poderiam ser abordados pelos ACS, a fim de desmistificar as informações imbuídas ao preventivo. Contudo, necessitam de conhecimentos prévios para ajudar a população, o que infelizmente nesse estudo se encontrou prejudicado, a exemplo de apenas 30,3\% saberem rastrear tal câncer.

Em relação ao câncer de mama, o presente estudo identificou que poucos ACS sabiam rastrear de forma adequada (27,27\%), resultado semelhante ao estudo de Prolla, Silva, Netto, Goldim e Ashton-Prolla (2015), que avaliou o conhecimento sobre o câncer de mama entre enfermeiros, identificando áreas de aprendizado deficientes principalmente nos assuntos que abordam fatores de risco e estratégias atuais para detecção do câncer, o que se cogita ser uma falha presente em alguns profissionais da saúde. Além disso, os participantes de seu artigo tiveram menor desempenho em perguntas relacionadas à ocorrência de câncer de mama em homens e hereditariedade (Prolla et al., 2015). No presente estudo a maioria dos voluntários relacionou a ocorrência da patologia a ambos os sexos $(83,33 \%)$, porém não sendo verificado se reconheciam algum grau de risco de hereditariedade quando acometido em homens.

Por outro lado, reforçando a necessidade dos profissionais conhecerem os fatores de risco para atuarem de forma precoce na conscientização, Silva e Riul (2011) mostraram que a maioria dos voluntários em quimioterapia para neoplasia de mama tinham dieta rica em gordura animal e fibras, estavam acima do peso e metade era tabagista. Nesse estudo, os mesmos fatores de risco/proteção são apontados pelos ACS em 86,4\%, 60,6\% (alimentação pobre em frutas), 78,8\% e 97\%, respectivamente. É válido ressaltar que os autores evidenciaram que quanto maior a escolaridade mais correta é a prática do autoexame das mamas, assim como maior a adesão ao exame de mamografia (Silva \& Riul, 2011). Dessa forma, percebe-se a necessidade de profissionais da saúde para informar a população com níveis educacionais mais baixos, principalmente para a realização do rastreio.

Quanto ao câncer de próstata, Gomes, Izidoro, e da Mata (2015) analisaram uma população de 92 trabalhadores de uma universidade, verificando que a principal fonte de informação era a televisão e rádio, e em menor proporção profissionais da saúde não médicos; uma parcela dos entrevistados só vão ao urologista quando sentem algo errado; e menos da metade (31.2\%) dos participantes mencionaram que não realizam a prevenção (exame de toque retal e o PSA) por ausência de sintomas relacionados à doença. A partir disso, percebe-se a necessidade de capacitar o ACS para dar informação à comunidade e orientar a ida do indivíduo às consultas na unidade de AB para investigação de sinais e sintomas. Nesse artigo os voluntários apresentaram conhecimentos equivocados quanto às principais sintomatologias iniciais do câncer de próstata, sendo conferido a ele aumento testicular $(57,58 \%)$ e mudança de hábitos intestinais (18.18\%). Ademais, como não há evidência científica de que o rastreamento do câncer de próstata traz mais benefícios do que riscos (INCA Câncer de próstata, 2019) se analisou apenas os sinais e sintomas.

Por fim, indivíduos que tiveram algum familiar com câncer apresentaram uma taxa de acerto significativa. Ao verificar a repercussão do diagnóstico de câncer no contexto familiar, estudos realizados com famílias de pacientes com câncer identificaram que os mesmos foram afetados pelo diagnóstico e acompanhamento do processo de adoecimento do familiar, principalmente por ser inesperado e carregar um estigma junto (Ferreira \& Dupas, 2016; Mattos, Blomer, Campos \& Silvério, 
2016). Contudo, Ferreira e Dupas (2016) mencionam a busca de informações sobre a doença como estratégia de enfrentamento para a família. Neste caso, favorecendo um maior conhecimento sobre o tema, o que explicaria o resultado do presente estudo. Entretanto, Mattos et al. (2016) mostra também que familiares podem passar pela situação evitando de falar sobre a doença tanto com o paciente quanto com outras pessoas, sendo uma forma de lidar com estresse, evitando o agente estressor. Em ambos os casos, espera-se que o familiar tenha mais informações que pessoas que não passaram por tal situação, principalmente pelo impacto gerado pelo diagnóstico. Infelizmente, este estudo não verificou se o voluntário tinha histórico pessoal de câncer, o que possivelmente também refletiria na quantidade de acertos.

Em vista disso, destaca-se a importância de avaliar o conhecimento dos profissionais de saúde e com os resultados desenvolver projetos de capacitação continuada, preenchendo as deficiências do conhecimento sobre doenças oncológicas evidenciadas no presente estudo. Assim, tais profissionais estariam mais aptos a atuarem na prevenção e detecção precoce de câncer na $\mathrm{AB}$. Além de orientarem sobre o fluxo do atendimento e tratamento em oncologia no nível primário de atenção. Desse modo, um paralelo pode ser feito com o estudo de Araújo, Almeida, Sá e Moura (2017) sobre o papel do ACS no controle da tuberculose e hanseníase, agindo na prevenção e vigilância, mas para tanto sendo necessária capacitações na formação dos ACS para garantir atuação qualificada na promoção da saúde.

A exemplo de ações de prevenção e controle do câncer cérvico-uterino na $\mathrm{AB}$, percebe-se que são realizadas em sua maioria por enfermeiros não devendo estar restrito apenas a eles, mas compartilhadas e redirecionadas com outros profissionais da AB, tais como os ACS, visando fortalecer a promoção da saúde (Silva, Rodrigues, Oliveira \& Melo, 2017).

Sendo assim, nosso estudo evidenciou a necessidade de constantes atualizações dos profissionais de saúde da AB, em especial os ACS. Pois participam da interlocução entre a unidade de saúde e a população assistida por ela, podendo facilitar a adesão da comunidade a realização de exames de rastreio e deteç̧ão precoce de sinais e sintomas relacionados ao câncer, com visitas regulares à unidade de saúde.

\section{Conclusão}

O presente estudo indica a possibilidade de deficiências no conhecimento dos ACS sobre doenças oncológicas na AB e seus níveis especializados. Contudo, tal trabalho apresenta limitações quanto a metodologia usada, amostra por conveniência e questionário não validado previamente. Além disso, reconhece-se a carência em diferenciar os entrevistados que apresentaram histórico de câncer. Em vista disso, percebe-se a necessidade de maiores trabalhos nesta temática.

Além disso, vale ressaltar que o pioneirismo do presente estudo servirá de suporte teórico e técnico para novas pesquisas científicas nessa área, uma vez que apresenta o cenário real da $\mathrm{AB}$, tanto as suas limitações quanto os seus pontos fortes, no enfrentamento ao câncer.

\section{Agradecimentos}

Agradecemos aos agentes comunitários de saúde que aceitaram participar e aos gestores das unidades de saúde da família que permitiram a execução deste trabalho.

\section{Referências}

Almeida B. (2013). O papel do agente comunitário de saúde no câncer de colo de útero [Monografia]. Uberaba (MG): Universidade Federal de Alfenas.

Amaral, A. F., Araújo, E. S., Magalhães, J. C., Silveira, E. A. D., Tavares, S. B. D. N. \& Amaral, R. G. (2014). Impacto da capacitação dos profissionais de saúde sobre o rastreamento do câncer do colo do útero em unidades básicas de saúde. Rev Bras Ginecol Obstet, 36(4):182-7.

American Cancer Society. 01 de setembro 2019. Cancer Facts \& Figures 2019. Atlanta: American Cancer Society.: https://azskincancerinstitute.org/sites/default/files/acs_facts_and_figures.pdf 
Araújo, E. T. H., Almeida, C. A. P. L., Sá, G. G. M. \& Moura, L. K. B. (2017). Produção científica da formação e atuação do agente comunitário de saúde sobre hanseníase e tuberculose. Rev Pre Infec e Saúde, 3(4), 46-56.

Brasil, Ministério da Saúde, Secretaria de Atenção à Saúde, Departamento de Atenção Básica. Política Nacional de Atenção Básica. Brasília: Ministério da Saúde, 2012, 1-110.

Brasil. Portaria n. ${ }^{\circ}$ 2.436, de 21 de setembro de 2017.Aprova a Política Nacional de Atenção Básica, estabelecendo a revisão de diretrizes para a organização da Atenção Básica, no âmbito do Sistema Único de Saúde (SUS). Diário Oficial da União 2017, 21 set.

Brito, L. M. (2016). O agente comunitário de saúde na prevenção do câncer: ressignificando o papel deste trabalhador. Revista Espaço Ciência \& Saúde, 4(1), $1-15$

Dantas, A. S., Moura, S. G., Albuquerque, K. F., Gerônimo, V. S., Andrade, J. H. \& Dantas, M. S. (2018). Representações sociais de agentes comunitários de saúde acerca do câncer de próstata. Revista de pesquisa Cuidado é fundamental online, 10 (1), 145-152.

Ferreira, M. L. S. M. \& Dupas, G. (2016). Repercussão do diagnóstico do câncer de mama no contexto familiar. Revista Brasileira de Pesquisa em Saúde/Brazilian Journal of Health Research, 18(4), 84-92.

Gomes, A. F. (2011). O papel do Agente Comunitário de Saúde na prevenção e detecção precoce do câncer de colo de útero [Monografia]. Campos Gerais (MG): Universidade Federal de Minas Gerais.

Gomes, C. R. G., Izidoro, L. C. R., \& da Mata, L. R. F (2015). Fatores de risco para o câncer de próstata e aspectos motivacionais e dificultadores na realização de práticas preventivas. Investigación Y Educación En Enfermería, 33 (3). https://revistas.udea.edu.co/index.php/iee/article/view/24449.

Hanahan, D. \& Weinberg, R. A. (2011). Hallmarks of cancer: the next generation. Cell, 144(5), 646-674.

Instituto Nacional De Câncer [homepage na internet]. Câncer de Próstata. https://www.inca.gov.br/tipos-de-cancer/cancer-de-prostata/profissional-de-saude.

Instituto Nacional De Câncer INCA. (2019). Onde Tratar Pelo Sus. https://www.inca.gov.br/onde-tratar-pelo-sus/para.

Instituto Nacional de Câncer José Alencar Gomes da Silva INCA. Rio de Janeiro, 2019. Estimativa 2020: incidência de câncer no Brasil / Instituto Nacional de Câncer José Alencar Gomes da Silva. p. 120.

Instituto Nacional de Câncer José Alencar Gomes da Silva INCA. (2020). ABC do câncer: abordagens básicas para o controle do câncer. Rio de Janeiro: Instituto Nacional de Câncer José Alencar Gomes da Silva. (6a ed.), rev. atualizada, p.13.

Manoel, A. L., Rodrigues, A. B., Piva, E. Z., Warpechowski, T. P. \& Schuelter-Trevisol F. (2017). Avaliação do conhecimento sobre o vírus do papiloma humano (HPV) e sua vacinação entre agentes comunitários de saúde na cidade de Tubarão, Santa Catarina, em 2014. Epidemiol. Serv. Saúde, Brasília, 26(2), 399-404.

Mattos, K., Blomer, T. H., Campos, A. C. B. F \& Silvério, M. R. (2016). Estratégias de enfrentamento do câncer adotadas por familiares de indivíduos em tratamento oncológico. Revista Psicologia e Saúde.

Ministério da Saúde. Portaria $n^{\circ}$ 140, de 27 de fevereiro de 2014. Redefine critérios e parâmetros para organização, planejamento, monitoramento, controle e avaliação dos estabelecimentos de saúde habilitados na atenção especializada em oncologia e define as condições estruturais, de funcionamento e de recursos humanos para a habilitação destes estabelecimentos no âmbito do SUS. Diário oficial da União, 2014.

Pereira, A., Shitsuka, D., Parreira, F., \& Shitsuka, R. (2018). Metodologia da pesquisa científica. Núcleo de Tecnologia Educacional NTE. https://repositorio.ufsm.br/bitstream/handle/1/15824/Lic_Computacao_Metodologia-Pesquisa-Cientifica.pdf?sequence=1

Prolla, C. M. D., Silva, P. S., Netto, C. B. O., Goldim, J. R. \& Ashton-Prolla, P. (2015). Conhecimento sobre câncer de mama e câncer de mama hereditário entre enfermeiras de um hospital público. Revista Latino-Americana de Enfermagem, 23 (1), 90-97. https://doi.org/10.1590/0104-1169.0185.2529.

Rodrigues, B. C., Carneiro, A. C. M. O., Silva, T. L., Solá, A. C. N., Manzi, N. M., Schechtman, N. P., Magalhães, H. L. G. O. \& Dytz, J. L. G. (2012). Educação em Saúde para a Prevenção do Câncer Cérvico-uterino. Rev Bras Educ Med, 36 (1), 149-14.

Santos, A. C. S. \& da Silva Varela, C. D. (2016). Prevenção do câncer de colo uterino: motivos que influenciam a não realização do exame de Papanicolaou. Revista Enfermagem Contemporânea, 4(2).

Silva, A. B., Rodrigues, M. P., Oliveira, A. P. \& Melo, R. H. V. (2017). Prevenção do câncer cervicouterino: uma ação realizada pelos enfermeiros da estratégia saúde da família? Revista Ciência Plural, 3 (2), 99-114.

Silva, A. R. S., Alves, E. R. P., Barros, M. B. S. C., Bushatsky, M., Souto, C. M. M. R. \& Figueira Filho, A. S. S. (2011). Educação em saúde para detecção precoce do câncer de mama. Rev. RENE, 12, 952-959.

Silva, P. A. \& Riul, S. S. (2011). Câncer de mama: fatores de risco e detecção precoce. Revista Brasileira de Enfermagem, 64(6), 1016-1021. https://doi.org/10.1590/S0034-71672011000600005.

Stormo, A.R., Moura, L. \& Saraiya, M. (2014). Cervical Cancer-Related Knowledge, Attitudes, and Practices of Health Professionals Working in Brazil's Network of Primary Care Units. The Oncologist, 19, 375-382.

Vogelstein, B., Papadopoulos, N., Velculescu, V. E., Zhou, S., Diaz, L. A. \& Kinzler, K. W. (2013). Cancer genome landscapes. Science, 339(6127), 15461558 .

World Health Organization WHO. 01 de setembro 2019. Câncer. https://www.who.int/health-topics/cancer\#tab=tab_1. 